\title{
Neutrino Astronomy: An Update
}

\author{
Francis Halzen \\ Department of Physics and Wisconsin IceCube Particle Astrophysics Center, \\ University of Wisconsin-Madison \\ email: halzen@wipac.wisc.edu
}

\begin{abstract}
Detecting neutrinos associated with the still enigmatic sources of cosmic rays has reached a new watershed with the completion of IceCube, the first detector with sensitivity to the anticipated fluxes. In this review, we will briefly revisit the rationale for constructing kilometer-scale neutrino detectors and summarize the status of the field.
\end{abstract}

Keywords. Neutrinos, cosmic rays, astrophysics

\section{Introduction}

Soon after the 1956 observation of the neutrino (Reines, 1956), the idea emerged that it represented the ideal astronomical messenger. Neutrinos reach us from the edge of the Universe without absorption and with no deflection by magnetic fields. Neutrinos have the potential to escape unscathed from the inner neighborhood of black holes, and, the subject of this update, from the cosmic accelerators where cosmic rays are born. Their weak interactions also make cosmic neutrinos very difficult to detect. Immense particle detectors are required to collect cosmic neutrinos in statistically significant numbers (Klein, 2008). Already by the 1970s, it had been understood that a kilometer-scale detector was needed to observe the "cosmogenic" neutrinos produced in the interactions of cosmic rays with background microwave photons (Roberts, 1992).

Today's estimates of the sensitivity for observing potential cosmic accelerators such as Galactic supernova remnants, active galactic nuclei (AGN), and gamma-ray bursts (GRB) unfortunately point to the same exigent requirement (Gaisser, 1995). Building a neutrino telescope has been a daunting technical challenge.

Given the detector's required size, early efforts concentrated on transforming large volumes of natural water into Cherenkov detectors that catch the light emitted by the secondary particles produced when neutrinos interact with nuclei in or near the detector (Markov, 1960). After a two-decade-long effort, building the Deep Underwater Muon and Neutrino Detector (DUMAND) in the sea off the main island of Hawaii unfortunately failed (Babson, 1990). However, DUMAND pioneered many of the detector technologies in use today and inspired the deployment of a smaller instrument in Lake Baikal (Balkanov, 2003) as well as efforts to commission neutrino telescopes in the Mediterranean (Aggouras, 2005; Aguilar, 2006; Migneco, 2008). These have paved the way toward the planned construction of KM3NeT.

The first telescope on the scale envisaged by the DUMAND collaboration was realized instead by transforming a large volume of transparent natural deep Antarctic ice into a particle detector, the Antarctic Muon and Neutrino Detector Array (AMANDA). In operation from 2000 to 2009, it represented the proof of concept for the kilometer-scale neutrino observatory, IceCube (ICPDD, 2001; Ahrens, 2004).

Neutrino astronomy has already achieved spectacular successes: neutrino detectors have "seen" the Sun and detected a supernova in the Large Magellanic Cloud in 1987. Both observations were of tremendous importance; the former showed that neutrinos 
have a tiny mass, opening the first crack in the Standard Model of particle physics, and the latter confirmed the basic nuclear physics of the death of stars. Fig. 1 illustrates the cosmic neutrino energy spectrum covering an enormous range, from microwave energies $\left(10^{-12} \mathrm{eV}\right)$ to $10^{20} \mathrm{eV}$ (Becker, 2008). The figure is a mixture of observations and theoretical predictions. At low energy the neutrino sky is dominated by neutrinos produced in the Big Bang. At MeV energy, neutrinos are produced by the sun and by supernova explosions; the flux from the 1987 event is shown. At higher energies the neutrino sky is dominated by neutrinos produced by cosmic-ray interactions in the atmosphere, measured up to energies of $100 \mathrm{TeV}$ by the AMANDA experiment (Achterberg, 2007). Atmospheric neutrinos are a key to our story, because they are the dominant background for extraterrestrial searches. The flux of atmospheric neutrinos falls dramatically with increasing energy; events above $100 \mathrm{TeV}$ are rare, leaving a clear field of view of the sky for extraterrestrial sources.

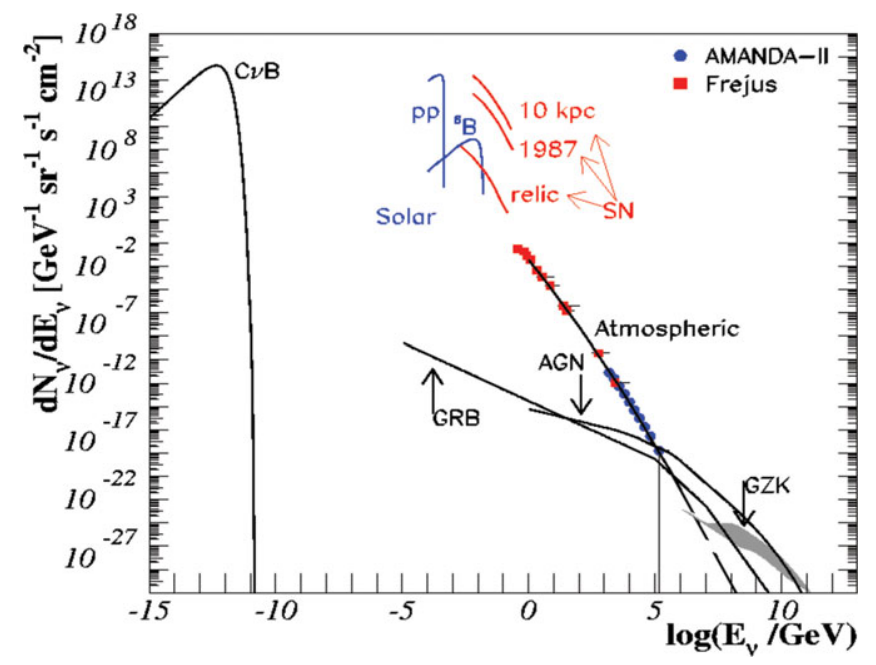

Figure 1. The cosmic-neutrino spectrum. Sources are the big bang $(\mathrm{C} \nu \mathrm{B})$, the Sun, supernovae $(\mathrm{SN})$, atmospheric neutrinos, gamma-ray bursts, active galactic nuclei, and cosmogenic (GZK) neutrinos. The data points are from a detector at the Fréjus underground laboratory (Rhode, 1996) (red) and from AMANDA (Achterberg, 2007) (blue).

The highest energy neutrinos in Fig. 1 are the decay products of pions produced by the interactions of cosmic rays with microwave photons (Ahlers, 2010). Above a threshold of $\sim 4 \times 10^{19} \mathrm{eV}$, cosmic rays interact with the microwave background introducing an absorption feature in the cosmic-ray flux, the Greisen-Zatsepin-Kuzmin (GZK) cutoff. As a consequence, the mean free path of extragalactic cosmic rays propagating in the microwave background is limited to roughly 75 megaparsecs. Therefore, the secondary neutrinos are the only probe of the still-enigmatic sources at further distances. The calculation of the neutrino flux associated with the observed flux of extragalactic cosmic rays is straightforward, and yields on the order of one event per year in a kilometerscale detector. The flux, labeled GZK in Fig. 1, shares the high-energy neutrino sky with neutrinos anticipated from gamma-ray bursts and active galactic nuclei (Gaisser, 1995).

\section{The First Kilometer-Scale Neutrino Detector: IceCube}

A series of first-generation experiments (Spiering, 2009; Katz, 2011) have demonstrated that high-energy neutrinos with $\sim 10 \mathrm{GeV}$ energy and above can be detected by observing 
Cherenkov radiation from secondary particles produced in neutrino interactions inside large volumes of highly transparent ice or water instrumented with a lattice of photomultiplier tubes. Construction of the first second-generation detector, IceCube, at the geographic South Pole was completed in December 2010 (Klein, 2010); see Fig. 2.

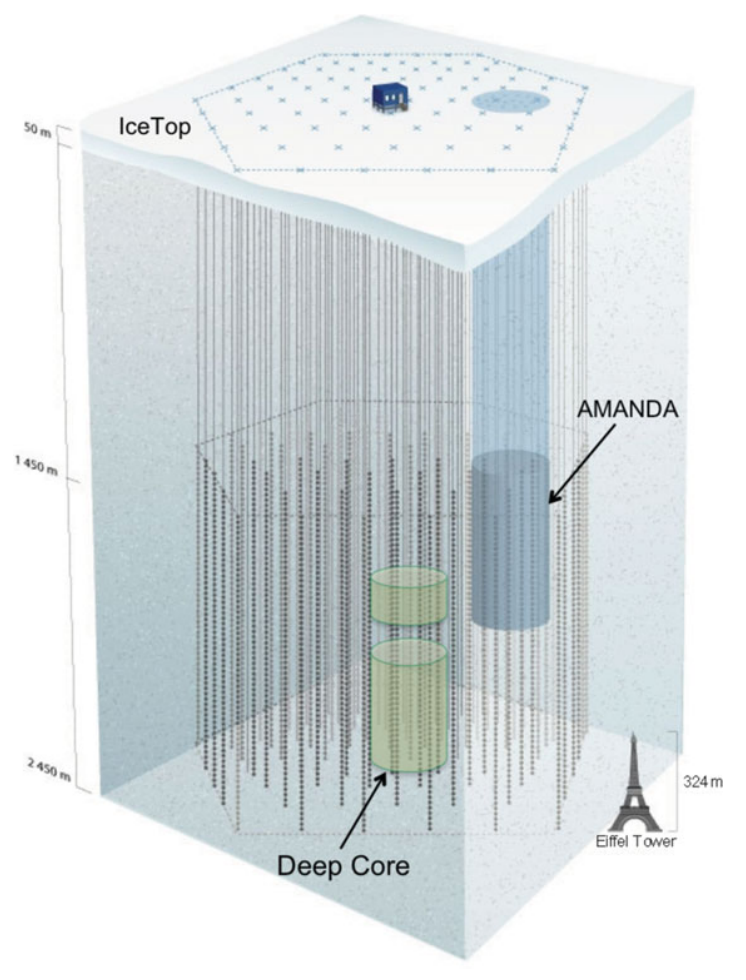

Figure 2. Schematic of the IceCube detector.

IceCube consists of 80 strings, each instrumented with 60 teninch photomultipliers spaced $17 \mathrm{~m}$ apart over a total length of one kilometer. The deepest module is located at a depth of $2.45 \mathrm{~km}$ so that the instrument is shielded from the large background of cosmic rays at the surface by approximately $1.5 \mathrm{~km}$ of ice. Strings are arranged at apexes of equilateral triangles that are $125 \mathrm{~m}$ on a side. The instrumented detector volume is a cubic kilometer of dark, highly transparent and sterile Antarctic ice. Radioactive background is dominated by the instrumentation deployed into this natural ice.

Each optical sensor consists of a glass sphere containing the photomultiplier and the electronics board that digitizes the signals locally using an onboard computer. The digitized signals are given a global time stamp with residuals accurate to less than 3 ns and are subsequently transmitted to the surface. Processors at the surface continuously collect the time-stamped signals from the optical modules, each of which functions independently. The digital messages are sent to a string processor and a global event builder. They are subsequently sorted into the Cherenkov patterns emitted by secondary muon tracks, or electron and tau showers, that reveal the direction of the parent neutrino (Halzen, 2006).

Based on data taken during construction, the actual effective area of the completed IceCube detector is increased by a factor $2(3)$ at $\mathrm{PeV}(\mathrm{EeV})$ energy over what had been expected (Ahrens, 2004). The neutrino collecting area is expected to increase with improved calibration and development of optimized software tools for the final detector, which has been operating stably in its final configuration since May 2011. Already reaching an angular resolution of better than 0.5 degree for muon tracks, this resolution can be reduced off-line to $\leqslant 0.2$ degree for individual events. The absolute pointing has been determined by measuring the shadowing of cosmic ray muons by the moon to 0.1 degree.

IceCube detects $10^{11}$ muons per year at a trigger rate of $2,700 \mathrm{~Hz}$. Among these it filters $10^{5}$ neutrinos, one every 6 minutes, above a threshold of $\sim 100 \mathrm{GeV}$. The DeepCore infill array identifies a similar sample with energies as low as $10 \mathrm{GeV}$; see Fig. 2. These muons and neutrinos are overwhelmingly of atmospheric origin and are the decay products of pions and kaons produced by collisions of cosmic-ray particles with nitrogen and oxygen 
in the atmosphere. Atmospheric neutrinos are a background for cosmic neutrinos, at least at energies below $100 \mathrm{TeV}$ where their flux cuts off sharply; see Fig. 3. At the highest energies, a small charm component is anticipated; its magnitude is uncertain and remains to be measured. As in conventional astronomy, IceCube must look through the atmosphere for cosmic neutrinos.

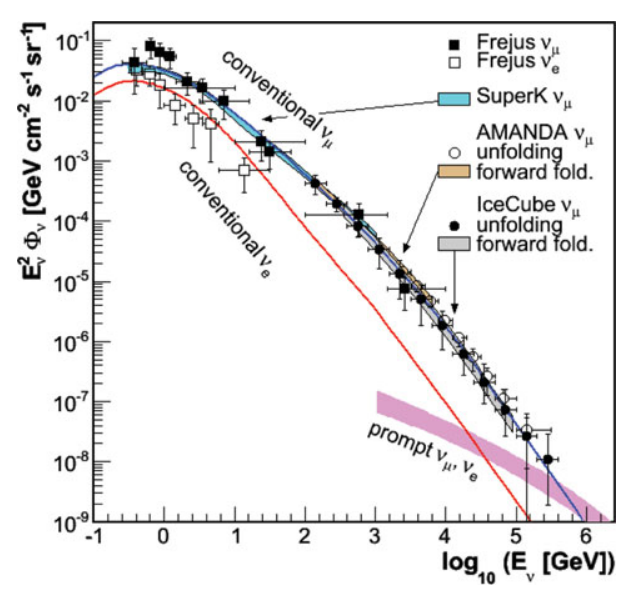

Figure 3. Measurements of the atmospheric neutrino energy spectrum; the Fréjus results (Daum, 1995), SuperK (Gonzalez-Garcia, 2006), AMANDA forward folding analysis (Abbasi, 2009) and unfolding analysis (Abbasi, 2010), and IceCube (40 strings) forward folding analysis (Abbasi, 2011b) and unfolding analysis (Abbasi, 2011a). All measurements include the sum of neutrinos and antineutrinos. The expectations for conventional $\nu_{\mu}$ and $\nu_{e}$ flux are from Barr, 2004. The prompt flux is from Enberg, 2008.

\section{Two Cosmic-Ray Puzzles}

Despite their discovery potential touching a wide range of scientific issues, the construction of groundbased gamma-ray telescopes and kilometer-scale neutrino detectors has been largely motivated by the possibility of opening a new window on the Universe in the TeV energy region, and above in the case of neutrinos. In this review we will revisit the prospects for detecting gamma rays and neutrinos associated with cosmic rays, thus revealing their sources at a time when we are commemorating the 100th anniversary of their discovery by Victor Hess in 1912 .

Cosmic accelerators produce particles with energies in excess of $10^{8} \mathrm{TeV}$; we still do not know where or how (Sommers, 2009). The flux of cosmic rays observed at Earth is shown in Fig. 4. The energy spectrum follows a sequence of three power laws. The first two are separated by a feature dubbed the "knee" at an energy $\dagger$ of approximately $3 \mathrm{PeV}$. There is evidence that cosmic rays up to this energy are Galactic in origin. Any association with our Galaxy disappears in the vicinity of a second feature in the spectrum referred to as the "ankle"; see Fig. 4. Above the ankle, the gyroradius of a proton in the Galactic magnetic field exceeds the size of the Galaxy, and we are almost certainly witnessing the onset of an extragalactic component in the spectrum that extends to energies beyond $100 \mathrm{EeV}$. Support for this assumption now comes from three experiments (Abbasi, 2008) that have observed the telltale structure in the cosmic-ray spectrum resulting from the absorption of the particle flux by the microwave background, the so-called Greisen-Zatsepin-Kuzmin (GZK) cutoff. Neutrinos are produced in GZK interactions; it was already recognized in the 1970s that their observation requires kilometer-scale neutrino detectors. The origin of the cosmic-ray flux in the intermediate region covering $\mathrm{PeV}$-to-EeV energies remains a mystery, although it is routinely assumed that its origin is some mechanism extending the reach of Galactic accelerators.

Acceleration of protons (or nuclei) to $\mathrm{TeV}$ energy and above requires massive bulk flows of relativistic charged particles. These are likely to originate from exceptional gravitational forces in the vicinity of black holes or neutron stars. The gravity of the collapsed

$\dagger$ We will use energy units $\mathrm{TeV}, \mathrm{PeV}$ and $\mathrm{EeV}$, increasing by factors of 1000 from GeV energy. 


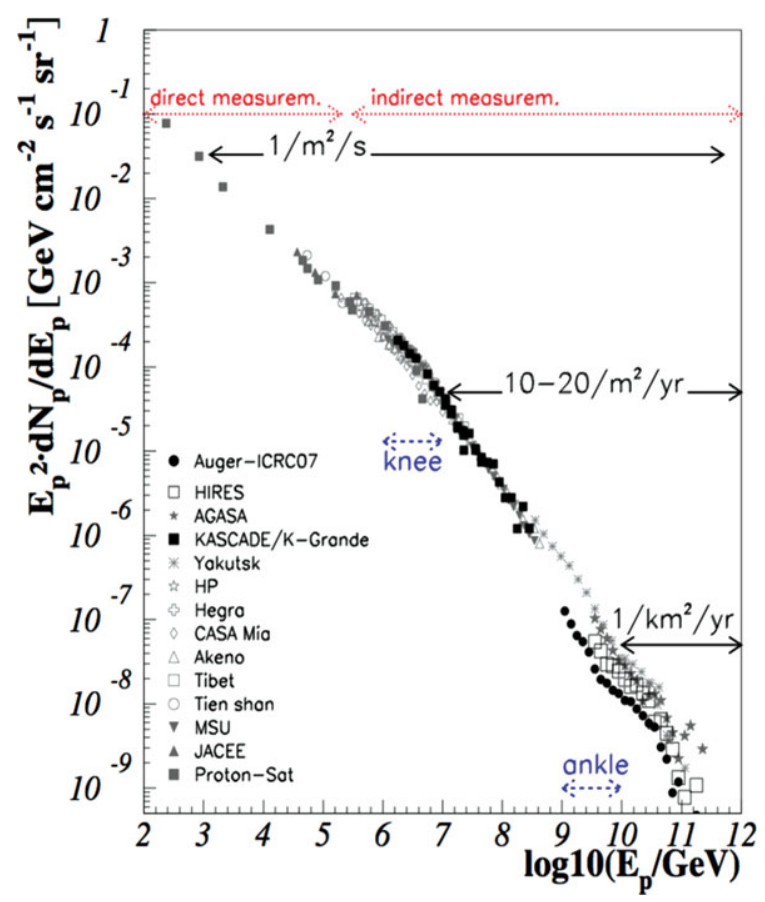

Figure 4. At the energies of interest here, the cosmic-ray spectrum follows a sequence of three power laws. The first two are separated by the "knee," the 2nd and 3rd by the "ankle." Cosmic rays beyond the ankle are a new population of particles produced in extragalactic sources.

objects powers large currents of charged particles that are the origin of high magnetic fields. These create the opportunity for particle acceleration by shocks. It is a fact that electrons are accelerated to high energy near black holes; astronomers detect them indirectly by their synchrotron radiation. Some cosmic sources must accelerate protons, because we observe them as cosmic rays.

The detailed blueprint for a cosmic-ray accelerator must meet two challenges: the highest energy particles in the beam must reach above $10^{3} \mathrm{TeV}$ for Galactic sources $\left(10^{8} \mathrm{TeV}\right.$ for extragalactic) and meet the total energy (luminosity) requirement to accommodate the observed cosmic-ray flux. Both represent severe constraints that have limited the imagination of theorists.

Supernova remnants were proposed as possible sources of Galactic cosmic rays as early as 1934 by Baade and Zwicky (Baade, 1934); their proposal is still a matter of debate after more than 70 years (Butt, 2009). Galactic cosmic rays reach energies of at least several PeV, the "knee" in the spectrum. Their interactions with Galactic hydrogen in the vicinity of the accelerator should generate gamma rays from the decay of secondary pions that reach energies of hundreds of $\mathrm{TeV}$. Such sources should be identifiable by a relatively flat energy spectrum that extends to hundreds of $\mathrm{TeV}$ without attenuation; they have been dubbed PeVatrons. The search to pinpoint them has so far been unsuccessful.

Although there is no incontrovertible evidence that supernovae accelerate cosmic rays, the idea is generally accepted because of energetics: three Galactic supernova explosions per century converting a reasonable fraction of a solar mass into particle acceleration can accommodate the steady flux of cosmic rays in the Galaxy. Energetics also drives speculations on the origin of extragalactic cosmic rays. 
By integrating the cosmic-ray spectrum in Fig. 4 above the ankle, we find that the energy density of the Universe in extragalactic cosmic rays is $\sim 3 \times 10^{-19} \mathrm{erg} \mathrm{cm}^{-3}$ (Gaisser, 1997). This value is rather uncertain because of our ignorance of the precise energy where the transition from Galactic to extragalactic sources occurs. The power required for a population of sources to generate this energy density over the Hubble time of $10^{10}$ years is $2 \times 10^{37} \mathrm{erg} \mathrm{s}^{-1}$ per $(\mathrm{Mpc})^{3}$. (In the astroparticle community, this flux is also known as $\left.5 \times 10^{44} \mathrm{TeV} \mathrm{Mpc}^{-3} \mathrm{yr}^{-1}\right)$.

A gamma-ray-burst fireball converts a fraction of a solar mass into the acceleration of electrons, seen as synchrotron photons. The observed energy in extragalactic cosmic rays can be accommodated with the reasonable assumption that shocks in the expanding GRB fireball convert roughly equal energy into the acceleration of electrons and cosmic rays (Waxman, 1995). It so happens that $2 \times 10^{51} \mathrm{erg}$ per GRB will yield the observed energy density in cosmic rays after $10^{10}$ years, given that their rate is on the order of 300 per $\mathrm{Gpc}^{3}$ per year. Hundreds of bursts per year over the Hubble time produce the observed cosmic-ray density, just like three supernovae per century accommodate the steady flux in the Galaxy.

Problem solved? Not really: it turns out that the same result can be achieved assuming that active galactic nuclei convert, on average, $2 \times 10^{44} \mathrm{erg} \mathrm{s}^{-1}$ each into particle acceleration. As is the case for GRBs, this is an amount that matches their output in electromagnetic radiation. Whether GRBs or AGN, the observation that these sources are required to radiate similar energies in photons and cosmic rays is unlikely to be an accident. We discuss the connection next; it will lead to a prediction of the neutrino flux.

\section{Neutrinos (and Photons) Associated with Cosmic Rays}

How many gamma rays and neutrinos are produced in association with the cosmic-ray beam? Generically, a cosmic-ray source should also be a neutrino beam dump. Cosmic rays accelerated in regions of high magnetic fields near black holes inevitably interact with radiation surrounding them. These may be photons radiated by the accretion disk in AGN and synchrotron photons that co-exist with protons in the exploding fireball producing a GRB. In these interactions, neutral and charged pion secondaries are produced by the processes

$$
p+\gamma \rightarrow \Delta^{+} \rightarrow \pi^{0}+p \text { and } p+\gamma \rightarrow \Delta^{+} \rightarrow \pi^{+}+n .
$$

While secondary protons may remain trapped in the high magnetic fields, neutrons and the decay products of neutral and charged pions escape. The energy escaping the source is therefore distributed among cosmic rays, gamma rays and neutrinos produced by the decay of neutrons, neutral pions and charged pions, respectively.

In the case of Galactic supernova shocks, cosmic rays inevitably interact with the hydrogen in the Galactic disk, producing equal numbers of pions of all three charges in hadronic collisions $p+p \rightarrow n\left[\pi^{0}+\pi^{+}+\pi^{-}\right]+X$; $n$ is the pion multiplicity. Their secondary fluxes should be boosted by the interaction of the cosmic rays with high-density molecular clouds that are ubiquitous in the star-forming regions where supernovae are more likely to explode.

In a generic cosmic beam dump, accelerated cosmic rays, assumed to be protons for simplicity, interact with a photon or proton target. In either case, accelerated cosmic rays produce charged and neutral pions. Subsequently, the pions decay into gamma rays and neutrinos that carry, on average, $1 / 2$ and $1 / 4$ of the energy of the parent pion. We here assume that the four leptons in the decay $\pi^{+} \rightarrow \nu_{\mu}+\mu^{+} \rightarrow \nu_{\mu}+\left(e^{+}+\nu_{e}+\bar{\nu}_{\mu}\right)$ equally share the charged pion's energy. The energy of the pionic leptons relative to the proton 
is:

$$
x_{\nu}=\frac{E_{\nu}}{E_{p}}=\frac{1}{4}\left\langle x_{p \rightarrow \pi}\right\rangle \simeq \frac{1}{20},
$$

and

$$
x_{\gamma}=\frac{E_{\gamma}}{E_{p}}=\frac{1}{2}\left\langle x_{p \rightarrow \pi}\right\rangle \simeq \frac{1}{10} .
$$

Here

$$
\left\langle x_{p \rightarrow \pi}\right\rangle=\left\langle\frac{E_{\pi}}{E_{p}}\right\rangle \simeq 0.2
$$

is the average energy transferred from the proton to the pion.

\section{Sources of the Extragalactic Cosmic Rays}

Waxman and Bahcall (Waxman, 1998) have presented an interesting benchmark for the neutrino flux expected from extragalactic cosmic ray accelerators, whatever they may be. The cosmic-ray flux, assuming an injected $E^{-2}$ spectrum before modification by absorption on the microwave background, can be parameterized as

$$
\frac{d N_{p}}{d E_{p}}=\frac{5 \times 10^{-11}}{E_{p}^{2}} \mathrm{TeV}^{-1} \mathrm{~cm}^{-2} \mathrm{~s}^{-1} \mathrm{sr}^{-1} .
$$

Integrating this flux, from the ankle to a maximal accelerator energy of $10^{9} \mathrm{TeV}$, accommodates the total energy requirement of $\sim 3 \times 10^{-19} \mathrm{erg} \mathrm{cm}^{-3}$. The secondary neutrino flux is given by

$$
\frac{d N_{\nu}}{d E}=\frac{1}{3}\left[\frac{2}{3}\right] \frac{1}{x_{\nu}} \frac{d N_{p}}{d E_{p}}\left(\frac{E}{x_{\nu}}\right) .
$$

Here the coefficients correspond to photo- and hadro-production of the neutrinos, respectively. $N_{\nu}\left(=N_{\nu_{\mu}}=N_{\nu_{e}}=N_{\nu_{\tau}}\right)$ represents the sum of the neutrino and antineutrino fluxes which are not distinguished by the experiments. Oscillations over cosmic baselines yield approximately equal fluxes for the three flavors. For the cosmic-ray flux introduced above, we obtain a neutrino flux

$$
\frac{d N_{\nu}}{d E} \simeq \frac{2 \times 10^{-12}}{E^{2}} \mathrm{TeV}^{-1} \mathrm{~cm}^{-2} \mathrm{~s}^{-1} \mathrm{sr}^{-1} .
$$

Notice that we sneaked in the assumption that each cosmic ray interacts once and only once in the target - if not, the flux is multiplied by the number of interactions $n_{\text {int }}$; see Halzen (2010) for a discussion. In fact, Waxman and Bahcall have argued that, if the density of the source were such that a high-energy cosmic ray interacted more than once, it would be opaque to $\mathrm{TeV}$ photons. So, the neutrino flux represents an upper limit for extragalactic sources that emit $\mathrm{TeV}$ gamma rays.

It is important to realize that the high-energy protons may be magnetically confined to the accelerator. In the case of GRBs, for instance, protons adiabatically lose energy, trapped inside the fireball that expands under radiation pressure until it becomes transparent and produces the display observed by astronomers. Secondary neutrons do escape with high energies and decay into protons that are the source of the observed extragalactic cosmic-ray flux. In this case, cosmic rays and pionic neutrinos are directly related by the fact that, for each secondary neutron decaying into a cosmic ray proton, there are 
three neutrinos produced by the associated $\pi^{+}$(see Eq. 3.1):

$$
E \frac{d N_{\nu}}{d E}=3 E_{n} \frac{d N_{n}}{d E_{n}}\left(E_{n}\right),
$$

and, after oscillations, per neutrino flavor

$$
E^{2} \frac{d N_{\nu}}{d E} \simeq\left(\frac{x_{\nu}}{x_{n}}\right) E_{n}^{2} \frac{d N_{n}}{d E_{n}}\left(E_{n}\right),
$$

where $x_{n} \sim 1 / 2$ is the relative energy of the secondary neutron; the neutron flux is identified with the observed cosmic ray flux. This straightforward prediction has been ruled out by IceCube with data taken during construction (Abbasi, 2012). There are alternative scenarios that, fortunately, also yield predictions within reach of the completed detector.

The key feature is that the normalization of the generic neutrino flux of Eq.5.2 is correct for GRBs because the fireball model generically predicts that $n_{\text {int }} \simeq 1$. The GRB phenomenology that successfully accommodates the astronomical observations, as well as the acceleration of cosmic rays, is that of the creation of a hot fireball of electrons, photons and protons that is initially opaque to radiation. The hot plasma therefore expands by radiation pressure, and particles are accelerated to a Lorentz factor $\Gamma$ that grows until the plasma becomes optically thin and produces the GRB display. The rapid time structure of the burst is associated with successive shocks (shells), of width $\Delta R=c \times t_{v}$, that develop in the expanding fireball. The rapid temporal variation of the radiation, $t_{v}$, is on the order of milliseconds, and can be interpreted as the collision of internal shocks with different Lorentz factors. Electrons, accelerated by first-order Fermi acceleration, radiate synchrotron gamma rays in the strong internal magnetic field, and thus produce the spikes observed in the emission spectra. The number of interactions of protons with the synchrotron photons is simply determined by the optical depth of the fireball shells of width $\Delta R$ to $\mathrm{p} \gamma$ interactions and is generically on the order of $n_{\text {int }} \simeq 1$.

Throughout the discussion of the neutrino flux associated with extragalactic cosmic rays we have neglected the fact that neutrinos, unlike cosmic rays, are not absorbed by microwave photons resulting in a neutrino flux increased by "a factor" that depends on the cosmological evolution of the sources. We have also assumed that the highest energy cosmic rays are protons. Experiments disagree on the composition but the cosmogenic flux is inevitably reduced in the presence of heavy primaries.

No compelling prediction is possible for AGN, complex systems with many possible sites for acceleration and interaction of the cosmic rays. Our discussion has, however, introduced the rationale that generic cosmic-ray sources produce a neutrino flux comparable to their flux of cosmic rays (Gaisser, 1997) and pionic TeV gamma rays (AlvarezMuniz, 2002). In this context we introduce Fig. 5 which shows the present IceCube upper limits on the neutrino flux from nearby AGN as a function of their distance. Also shown is the TeV gamma-ray emission from the same sources; except for CenA and M87, the muon-neutrino limits have reached the level of the $\mathrm{TeV}$ photon flux. This is a notable fact because of the equipartition of the cosmic-ray, gamma-ray and neutrino fluxes from a cosmic ray accelerator. One can sum the sources shown in the figure into a diffuse flux; the result is, after dividing by $4 \pi / c, 3 \times 10^{-12} \mathrm{TeV} \mathrm{cm}^{-2} \mathrm{~s}^{-1} \mathrm{sr}^{-1}$, or approximately $10^{-11} \mathrm{TeV} \mathrm{cm}^{-2} \mathrm{~s}^{-1} \mathrm{sr}^{-1}$ for all neutrino flavors. This flux matches the "maximal" flux previously argued for; see Fig. 3 .

IceCube's sensitivity is rapidly approaching this benchmark flux as shown in Fig. 6. In fact, the IceCube "limit" obtained with one year of data taken with 59 strings is a $1.8 \sigma$ signal (Sullivan, 2012). Although not significant, it is of interest that the benchmark flux argued for above rises above the atmospheric neutrino background for energies exceeding 


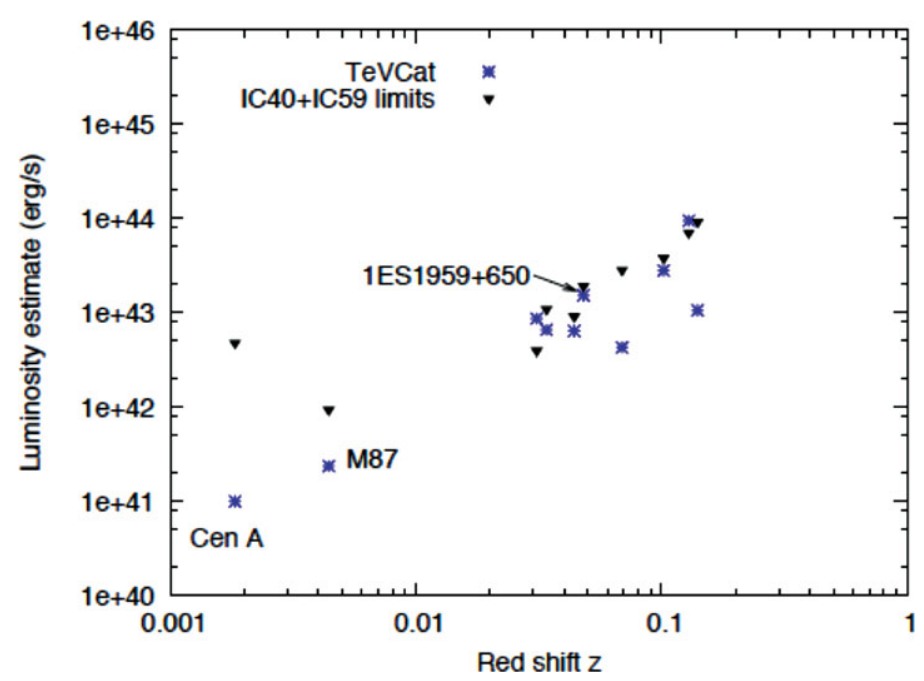

Figure 5. IceCube neutrino flux limits are compared with the TeV photon flux for nearby AGN. Figure courtesy of T. Gaisser.

$100 \mathrm{TeV}$ at a flux level already reached by the completed IceCube detector after one year of operation.

The improved performance of IceCube at EeV energy has created the opportunity to detect cosmogenic neutrinos. We anticipate 2.3 events in three years of running the completed detector assuming a flux derived from the "best fit" to the cosmic-ray data (Ahlers, 2010), and 4.8 events for the largest neutrino rate allowed by the constraint imposed on their accompanying photon flux by the observed flux of diffuse photons in the Universe (Ahlers, 2010).

Recently, in a dedicated search for cosmogenic neutrinos two events have been found (Ishihara, 2012) in the first year of data taken with the completed detector. They are showers of more than $10^{5}$ photons, about $500 \mathrm{~m}$ in diameter, fully contained inside the detector. With no evidence of a muon track, they are initiated by electron or tau neutrinos; see Fig. 7. However, their energies, rather than super-EeV as expected for cosmogenic neutrinos, are in the $\mathrm{PeV}$ range: 1.1 and $1.3 \mathrm{PeV}$ with a negligible statistical error and a $35 \%$ systematic error. The analysis of these events is ongoing and we expect this error to be significantly reduced in the near future. We have also determined the directions of the initial neutrinos exploiting the fact that the waveforms collected by the DOMs following and trailing the initial neutrino direction are identifiably different.

More importantly, we have designed a dedicated starting-event analysis to find more such events in the same data sample. Some of them should contain muon tracks whose arrival directions can be reconstructed with superior precision to that of the two shower events. The events are likely to represent new neutrino physics, or astrophysics, because their atmospheric origin is excluded, very conservatively, below the $10^{-2}$ level. Accommodating the events as the decay of charm particles produced in the atmosphere requires a flux that violates the IceCube diffuse limit obtained with data collected with 59 strings (Sullivan, 2012). 

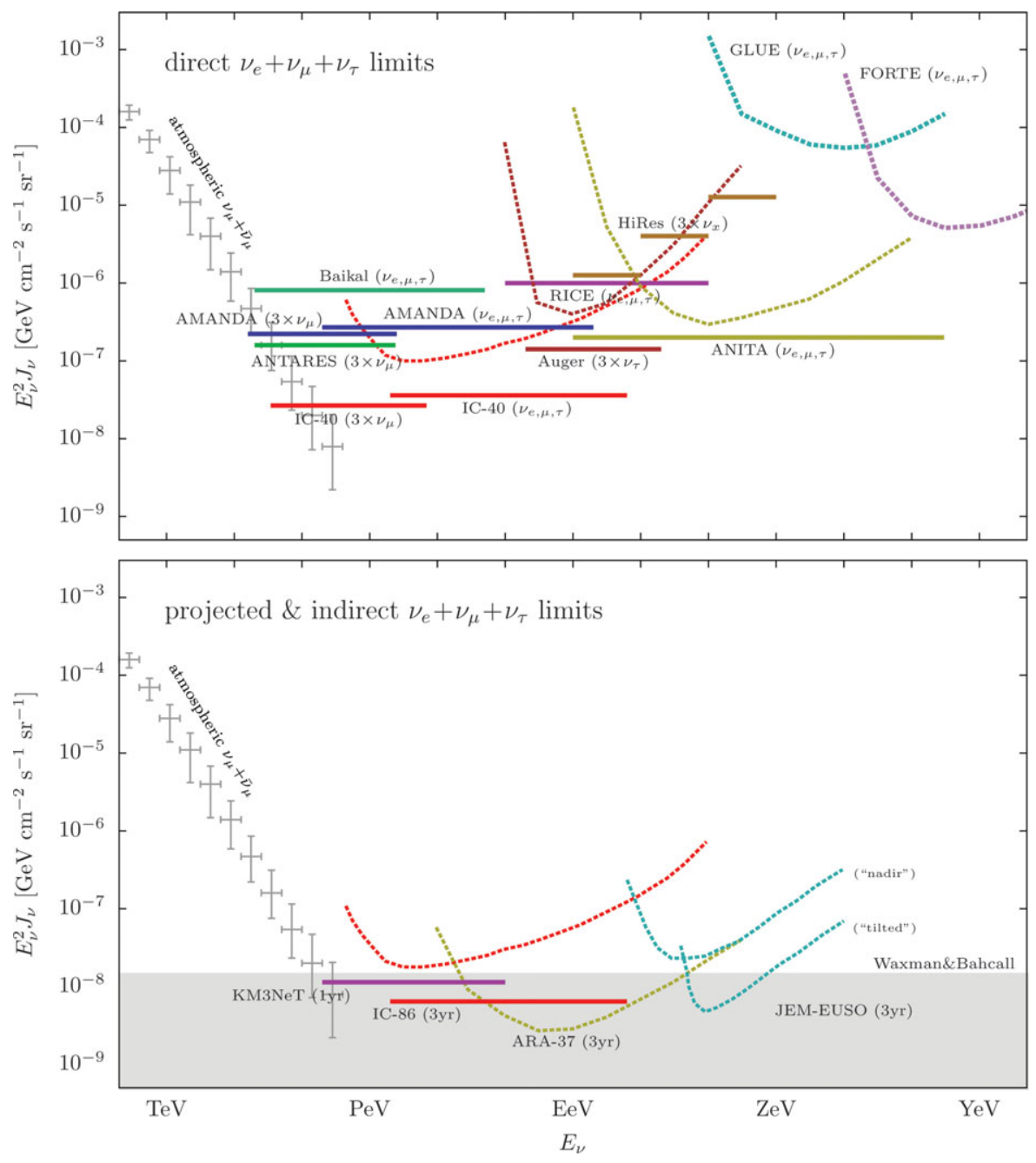

Figure 6. Limits on a diffuse neutrino flux from existing (top) and future (bottom) experiments; see Achterberg, 2007, et al. The shaded band indicates the anticipated neutrino fluxes associated with cosmic rays. Figure courtesy of M. Ahlers.

\section{Sources of Galactic Cosmic Rays}

Despite the commissioning of instruments with improved sensitivity, it has been impossible to conclusively pinpoint supernova remnants as the sources of the Galactic cosmic rays by identifying the accompanying gamma rays of pion origin. The position of the knee in the cosmic-ray spectrum indicates that some sources must accelerate cosmic rays to energies of several PeV. These so-called PeVatrons therefore produce pionic gamma rays whose spectrum extends to several hundred $\mathrm{TeV}$ without cutoff. In contrast, the widely studied supernova remnants RX J1713-3946 and RX J0852.0-4622 (Vela Junior) reach their maximum energy in the $\mathrm{TeV}$ region. In fact, recent data from Fermi LAT have directly challenged the hadronic interpretation of the $\mathrm{GeV}-\mathrm{TeV}$ radiation from one of the best-studied candidates, RX J1713-3946 (Abdo, 2011).

It is difficult to hide a Galactic cosmic accelerator from view. A generic supernova remnant releasing an energy of $W \sim 10^{50} \mathrm{erg}$ into the acceleration of cosmic rays will inevitably generate $\mathrm{TeV}$ gamma rays in the interaction of the accelerated nuclei with 

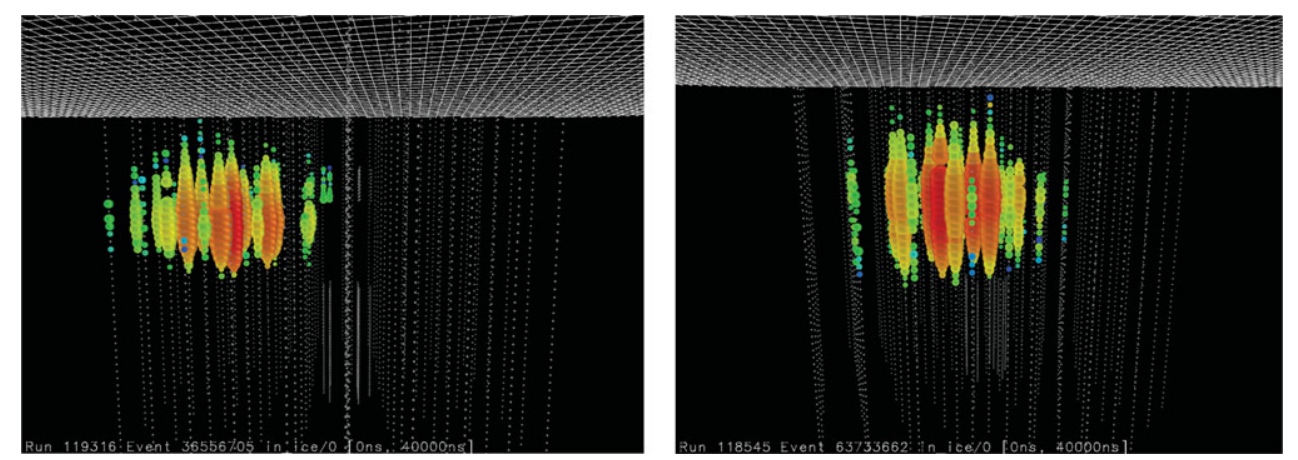

Figure 7. Displays of the two observed events. Each colored sphere represent a DOM that sent a time-stamped waveform to the event builder. Colors indicate the arrival timing of the photon (red=early, blue=late). The size of the sphere indicates the number of photons detected by each DOM.

the hydrogen in the Galactic disk. The emissivity in pionic gamma rays $Q_{\gamma}$ is simply proportional to the density of cosmic rays $n_{c r}$ and the density of the target $n \sim 1 / \mathrm{cm}^{3}$ of protons in the disk. Here $n_{c r}(>1 \mathrm{TeV}) \simeq 4 \times 10^{-14} \mathrm{~cm}^{-3}$ is obtained by integrating the proton spectrum for energies in excess of $1 \mathrm{TeV}$. For an $E^{-2}$ spectrum

$$
Q_{\gamma} \simeq c\left\langle\frac{E_{\pi}}{E_{p}}\right\rangle \lambda_{p p}^{-1} n_{c r}(>1 \mathrm{TeV}) \simeq 2 c x_{\gamma} \sigma_{p p} n n_{c r}
$$

or

$$
Q_{\gamma}(>1 \mathrm{TeV}) \simeq 10^{-29} \mathrm{TeV} \mathrm{cm}^{-3} \mathrm{~s}^{-1}\left(\frac{n}{1 \mathrm{~cm}^{-3}}\right) .
$$

The proportionality factor in Eq. (6.1) is determined by particle physics; $x_{\gamma} \simeq 0.1$ is the average energy of secondary photons relative to the cosmic ray protons and $\lambda_{p p}=$ $\left(n \sigma_{p p}\right)^{-1}$ is the proton interaction length $\left(\sigma_{p p} \simeq 40 \mathrm{mb}\right)$ in a density $n$. The corresponding luminosity is

$$
L_{\gamma}(>1 \mathrm{TeV}) \simeq Q_{\gamma} \frac{W}{\rho_{E}},
$$

where $W / \rho_{E}$ is the volume occupied by the supernova remnant. Here we made the approximation that the volume of the young remnant is given by $W / \rho_{E}$, or that the density of particles in the remnant is not very different from the ambient energy density $\rho_{E} \sim 10^{-12} \mathrm{erg} \mathrm{cm}^{-3}$ of Galactic cosmic rays.

We thus predict (Gonzalez-Garcia, 2009) a rate of $\mathrm{TeV}$ photons from a supernova at a nominal distance $d$ on the order of $1 \mathrm{kpc}$ of

$$
\begin{aligned}
& \int_{E>1 \mathrm{TeV}} \frac{d N_{\gamma}}{d E_{\gamma}} d E_{\gamma}=\frac{L_{\gamma}(>1 \mathrm{TeV})}{4 \pi d^{2}} \\
& \simeq 10^{-12}-10^{-11}\left(\frac{\text { photons }}{\mathrm{cm}^{2} \mathrm{~s}}\right)\left(\frac{W}{10^{50} \mathrm{erg}}\right)\left(\frac{n}{1 \mathrm{~cm}^{-3}}\right)\left(\frac{d}{1 \mathrm{kpc}}\right)^{-2} .
\end{aligned}
$$

This is a PeVatron flux well within reach of the current generation of atmospheric gamma ray telescopes; has it been detected?

Looking for them in the highest energy survey of the Galactic plane is evident and points to the Milagro experiment (Abdo, 2007). Their survey in the $\sim 10 \mathrm{TeV}$ band revealed a subset of sources located within nearby star-forming regions in Cygnus and in the vicinity of Galactic latitude $l=40$ degrees. Subsequently, directional air Cherenkov telescopes were pointed at three of the sources, revealing them as PeVatron candidates 
with an $E^{-2}$ energy spectrum that extends to tens of $\mathrm{TeV}$ without evidence of a cutoff (Djannati-Atai, 2007; Albert, 2008) and gamma ray fluxes in the range estimated above.

Interestingly, some of the sources cannot be readily associated with known supernova remnants, or with any non-thermal source observed at other wavelengths. These are likely to be molecular clouds illuminated by the cosmic-ray beam accelerated in young remnants located within about $100 \mathrm{pc}$. Indeed, one expects that multi-PeV cosmic rays are accelerated only over a short time period when the shock velocity is high, i.e., when the remnant transitions from free expansion to the beginning of the Sedov phase. The high-energy particles can produce photons and neutrinos over much longer periods when they diffuse through the interstellar medium to interact with nearby molecular clouds (Gabici, 2007). An association of molecular clouds and supernova remnants is expected, of course, in star-forming regions. In this case, any confusion with synchrotron photons is unlikely.

Particle physics dictates the relation between pionic gamma rays and neutrinos and basically predicts the production of a $\nu_{\mu}+\bar{\nu}_{\mu}$ pair for every two gamma rays seen by Milagro. This calculation can be performed using the formalism introduced in the previous section with approximately the same outcome.

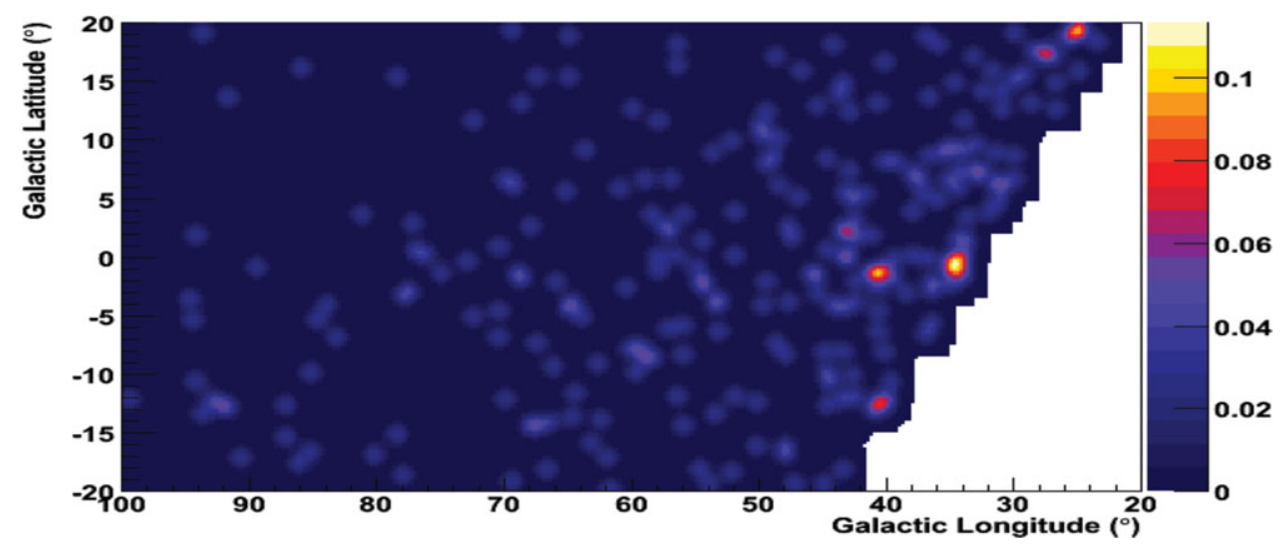

Figure 8. Simulated sky map of IceCube in Galactic coordinates after 5 years of operation of the completed detector. Two Milagro sources are visible with 4 events for MGRO J1852+01 and 3 events for MGRO J1908+06 with energy in excess of $40 \mathrm{TeV}$. These, as well as the background events, have been randomly distributed according to the resolution of the detector and the size of the sources.

The quantitative statistics can be summarized as follows. For average values of the parameters parametrizing the flux, we find that the completed IceCube detector should confirm sources in the Milagro sky map as sites of cosmic-ray acceleration at the $3 \sigma$ level in less than one year and at the $5 \sigma$ level in three years (Gonzalez-Garcia, 2009); see Fig. 8. This assumes that the source extends to $300 \mathrm{TeV}$, or $10 \%$ of the energy of the cosmic rays near the knee in the spectrum. These results agree with previous estimates (Halzen, 2008). There are intrinsic ambiguities of an astrophysical nature in this estimate that may reduce or extend the time required for a $5 \sigma$ observation (Gonzalez-Garcia, 2009). Also, the extended nature of some of the Milagro sources represents a challenge for IceCube observations that are optimized for point sources. In the absence of an observation of $\mathrm{TeV}$-energy supernova neutrinos by IceCube within a period of 10 years, the supernova origin of cosmic rays in the Galaxy will be challenged. 


\section{Conclusion: Stay Tuned}

In summary, IceCube was designed for a statistically significant detection of cosmic neutrinos accompanying cosmic rays in five years. Here we made the case that, based on multiwavelength information from ground-based gamma ray telescopes and cosmic-ray experiments, we are indeed closing in on supernova remnants, GRBs (if they are the sources of cosmic rays) and GZK neutrinos. The discussion brought to the forefront the critical role of improved spectral gamma-ray data on candidate cosmic-ray accelerators. The synergy between CTA (see CTA), IceCube, and KM3NeT as well as other nextgeneration neutrino detectors is likely to provide fertile ground for future progress.

\section{Acknowledgements}

This research was supported in part by the U.S. National Science Foundation under Grants No. OPP-0236449 and PHY-0969061; by the U.S. Department of Energy under Grant No. DE-FG02-95ER40896; by the University of Wisconsin Research Committee with funds granted by the Wisconsin Alumni Research Foundation.

\section{References}

Abbasi, R., et al. (HiRes Collaboration), 2008, Phys. Rev. Lett. 100, 101101, astro-ph/0703099 ; Abraham, J., et al. (Auger collaboration), 2008, Phys. Rev. Lett. 101, 061101, astroph/08064302; and Tokuno, H., et al., "The Status of the Telescope Array Experiment," J. Phys. Conf. Ser. 293:012035 (2011)

Abbasi, R., et al. (IceCube Collaboration), 2009, Phys. Rev. D 79102005

Abbasi, R., et al. (IceCube Collaboration), 2010, Astropart. Phys. 3448

Abbasi, R., et al. (IceCube Collaboration), 2011, Phys. Rev. D 83012001

Abbasi, R., et al. (IceCube Collaboration), 2011, Phys. Rev. D 84082001

Abbasi, R., et al. (IceCube Collaboration), 2012, Nature 484, 351; astro-ph.HE/1204.4219

Abdo, A. A., et al. (Fermi-LAT Collaboration), 2011, Astrophys. J. 734, 28; astroph.HE/1103.5727v1

Abdo, A. A., et al. (Milagro Collaboration), 2007, Astrophys. J. 658, L33; astro-ph/0611691

Achterberg, A., et al. (IceCube Collaboration), 2007 Phys. Rev. D 76, 042008, Erratum ibid. 77, 089904(E); astro-ph/07051315

See Achterberg, A., 2007 cited above; Ackermann, M., et al. (IceCube collaboration), 2008, Astrophys. J. 675 , 1014, astro.ph/0711.3022 ; Abbasi, R., et al. (IceCube collaboration), 2010, Phys. Rev. D 82, 072003, astro.ph.CO/1009.1442v1; Aynutdinov, V., et al. (Baikal collaboration), 2006, Astropart. Phys. 25, 140, astro-ph/0508675 ; Martens, K., et al. (HiRes collaboration), 2007, Proceedings of 23rd Lepton-Photon Conference, Daegu, Korea, astroph/0707.4417 ; Kravchenko, I., et al. (RICE collaboration), 2006, Phys. Rev. D 73, 082002, astro-ph/0601148 ; Barwick, S. W., et al. (ANITA collaboration), 2006, Phys. Rev. Lett. 96, 171101, astro-ph/0512265 ; Lehtinen, N. G., Gorham, P. W., Jacobson, A. R., \& RousselDupre, R. A., 2004, Phys. Rev. D 69, 013008, astro-ph/0309656 ; Gorham, P. W., Hebert, C. L., Liewer, K. M., Naudet, C. J., Saltzberg, D., \& Williams, D., 2004, Phys. Rev. Lett. 93, 041101, astro-ph/0310232 ; Anchordoqui, L. A., Feng, J. L., Goldberg, H., \& Shapere, A. D., 2002, Phys. Rev. D 66, 103002, hep-ph/0207139; and Abbasi, R., et al. (IceCube collaboration), 2011,Phys. Rev. D 83, 012001, astro-ph.HE/1010.3980

Aggouras, G., et al. (NESTOR Collaboration), 2005, Astropart. Phys. 23, 377

Aguilar, J. A., et al. (ANTARES Collaboration), 2006, Astropart. Phys. 26, 314

Ahlers, M., Anchordoqui, L. A., Gonzalez-Garcia, M. C., et al., 2010, Astropart. Phys. 34, 106; astro-ph.HE/1005.2620

Ahrens, J., et al. (IceCube Collaboration), 2004, Astropart. Phys. 20, 507, astro-ph/0305196

Albert, J. et al., 2008; astro-ph/08012391 
Alvarez-Muniz, J., \& Halzen, F., 2002, Astrophys. J. 576, L33, astro-ph/0205408; for a more recent discussion of the formalism, see Becker, J. K., Halzen, F., \& OMurchadha, A, Olivo, M., 2010, Astrophys. J. 721, 1891, astro-ph.HE/0911.2202

Baade, W. \& Zwicky, F., 1934, Phys. Rev. D 46, 76

Babson, J. Barish, R., Becker-Szendy, R., et al., 1990, Phys. Rev. D 42, 3613

Balkanov, V. A., et al. (BAIKAL Collaboration), 2003, Nucl. Phys. B Proc. Suppl. 118, 363

Barr, G. D., et al.., 2004, Phys. Rev. D 70 023006, 2004

Becker, J. K., 2008, Phys. Rep. 458, 173; astro-ph/07101557

Butt, Y., 2009, Nature 460, 701; astro-ph.he/10093664

Cherenkov Telescope Array (CTA), http://www.cta-observatory.org

Daum, K., et al., 1995, Zeitschrift für Physik C 66417

Djannati-Atai, A., et al. (H. E. S. S. Collaboration), 2007, 30th ICRC, Merida, Mexico OG2.2 2, 1316; astro-ph/07102418

Enberg, R., Reno, M. H., \& Sarcevic, I., 2008, Phys. Rev. D 78043005

Gabici, S., \& Aharonian, F. A., 2008, Astrophys. J. 665 2, 431; astro-ph/0705.3011

\&Gaisser, T. K., 1997, OECD Megascience Forum, Taormina, Italy, astro-ph/9707283 ; the discussion was revisited in Ahlers, M., Anchordoqui, L. A., \& Goldberg, H., et al., 2005, Phys. Rev. D 72, 023001, astro-ph/0503229

Gaisser, T. K., Halzen, F., \& Stanev, T., 1995, Phys. Rept., 258, 173, Erratum 271, 355, hepph/9410384 ; Learned, J. G., \& Mannheim, K., 2000, Ann. Rev. Nucl. Part. Science, 50, 679 ; Halzen, F., \& Hooper, D., 2002, Rep. Prog. Phys., 65 , 1025, astro-ph/0204527 ; and Katz, U. F., \& Spiering, C., 2012, Prog. Part. Nucl. Phys., 67, 651, astro-ph.HE/1111.0507

Gonzalez-Garcia, M. C., Maltoni, M., \& Rojo, J., 2006, J. High Energy Phys. 1075

Gonzalez-Garcia, M. C., Halzen, F., \& Mohapatra, S., 2009, Astropart. Phys. 31, 437; astroph.HE/09021176

Halzen, F., 2006, Eur. Phys. J. C 46, 669; astro-ph/0602132

Halzen, F., 2011, Acta Phys. Polon. B 42, 2525; arXiv:1111.1131 [hep-ph]

Halzen, F., Kappes, A., \& OMurchadha, 2008, Phys. Rev. D 78, 063004, see also Nucl. Instr. and Meth. A 602, 117 (2009); astro-ph/0803.0314v2

Halzen, F., \& Klein, S. R., 2008, Physics Today, 61N5, 29

Halzen, F. \& Klein, S. R., 2010, Rev. Sci. Instrum. 81, 081101; astro-ph.HE/1007.1247

IceCube Collaboration, 2001, available at http://www.icecube.wisc.edu/science/ publications/pdd/pdd.pdf

Ishihara, A., 2012, Proceedings of Neutrino 2012, Kyoto, Japan

Katz, U. \& Spiering, C., 2011, Prog. Part. Nucl. Phys. 67, 651; astro-ph.HE/1111.0507

Markov, M. A., 1960, Proc. 1960 Intl. Conf. on High Energy Physics 578

Migneco, E., 2008, J. Phys. Conf. Ser. 136, 022048

Reines, F. \& Cowan, Jr., C. L., 1956, Nature, 17, 446

Rhode, W., et al. (Fréjus Collaboration), 1996, Astropart Phys. 4, 217

Roberts, A., 1992, Rev. Mod. Phys., 64, 259

Sommers, P., \& Westerhoff, S., 2009, New J. Phys. 11, 055004, astro-ph/08021267 Hillas, A. M., 2006, astro-ph/0607109v2 ; and Berezinsky, V, 2008, J. Phys. Conf. Ser. 120, 012001, astro$\mathrm{ph} / 08013028$

Spiering, C., 2009, AIP Conf. Proc. 1085, 18; astro-ph/0811.4747

Sullivan, G., 2012, Proceedings of Neutrino 2012, Kyoto, Japan

Waxman, E., 1995, Phys. Rev. Lett. 75, 386, astro-ph/9701231 ; Vietri, M, 1998, Phys. Rev. Lett. 80, 3690, astro-ph/9802241 ; and Bottcher, M., \& Dermer, C. D., 1998, Astrophys. J. Lett. 499, L131, astro-ph/9801027v2

Waxman, E. \& Bahcall, J., 1998, Phys. Rev. D59, 023002 